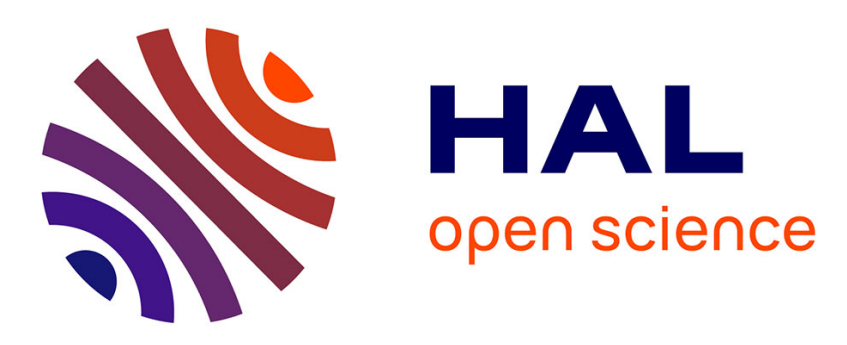

\title{
Games with distributionally robust joint chance constraints
}

Shen Peng, Abdel Lisser, Vikas Vikram Singh, Nalin Gupta, Eshan

Balashandar

\section{- To cite this version:}

Shen Peng, Abdel Lisser, Vikas Vikram Singh, Nalin Gupta, Eshan Balashandar. Games with distributionally robust joint chance constraints. Optimization Letters, inPress. hal-03106377

\section{HAL Id: hal-03106377 \\ https://hal-centralesupelec.archives-ouvertes.fr/hal-03106377}

Submitted on 11 Jan 2021

HAL is a multi-disciplinary open access archive for the deposit and dissemination of scientific research documents, whether they are published or not. The documents may come from teaching and research institutions in France or abroad, or from public or private research centers.
L'archive ouverte pluridisciplinaire HAL, est destinée au dépôt et à la diffusion de documents scientifiques de niveau recherche, publiés ou non, émanant des établissements d'enseignement et de recherche français ou étrangers, des laboratoires publics ou privés. 


\title{
Games with distributionally robust joint chance constraints
}

\author{
Shen Peng • Abdel Lisser • Vikas \\ Vikram Singh • Nalin Gupta • Eshan \\ Balachandar
}

Received: date / Accepted: date

\begin{abstract}
This paper studies an $n$-player non-cooperative game where each player has expected-value payoff function and chance-constrained strategy set. We consider the case where the row vectors defining the constraints are independent random vectors whose probability distributions are not completely known and belong to a certain distributional uncertainty set. The chanceconstrained strategy sets are defined using a distributionally robust framework. We consider one density based uncertainty set and four two-moments based uncertainty sets. One of the considered uncertainty sets is based on a nonnegative support. Under the standard assumptions on the players' payoff functions, we show that there exists a Nash equilibrium of a distributionally robust chance-constrained game for each uncertainty set. As an application, we study Cournot competition in electricity market and perform the numerical experiments for the case of two electricity firms.
\end{abstract}

Keywords Chance-constrained game · Nash equilibrium · Distributionally robust optimization · Nonnegative support · Electricity market.

Shen Peng

Department of Mathematics, KTH Royal Institute of Technology, Stockholm, Sweden

E-mail: shenp@kth.se

Abdel Lisser

Laboratoy of Signals and Systems, CentraleSupelec, Bat Breguet, 3 Rue Joliot Curie, 91190 Gif-sur-Yvette, France

E-mail: abdel.lisser@centralesupelec.fr

Vikas Vikram Singh

Department of Mathematics, IIT Delhi, New Delhi, India

E-mail: vikassingh@maths.iitd.ac.in

Nalin Gupta

Department of Mathematics, IIT Delhi, New Delhi, India

E-mail: nalingupta98@gmail.com

Eshan Balachandar

Department of Mathematics, IIT Delhi, New Delhi, India

E-mail: eshan.balachandar@gmail.com 


\section{Introduction}

The theory of games was started with the minimax theorem by John von Neumann 24] which establishes the existence of a saddle point equilibrium in a zero-sum game. Later John Nash [23] showed that there exists a mixed strategy equilibrium, which is called a Nash equilibrium, for a finite strategic non-cooperative game with finite number of players. Since then, the noncooperative strategic games have been extensively studied in the literature. The existence of a Nash equilibrium was shown under certain conditions on the strategy sets and payoff functions [2, 11, 13].

The above mentioned papers consider the games where players' strategy sets and payoff functions are deterministic in nature. In some practical game theoretic situations, the uncertainties are present due to various external factors. There are various ways to address the uncertainties present in the model. The robust optimization framework is used to handle distribution free uncertainties in the model 11, 34. For the uncertainties involving random variables, the expected payoff criterion is used in case of risk neutral players [15, 16, 19, 26, 35, 36] and the risk measures CVaR and variance are used in the risk averse case [10, 18, 26. For finite strategic games with random payoffs, Singh et al. [29, 30, 31] introduced a chance constraint programming based payoff criterion. It captures a situation where players are guaranteed to get the payoffs with a certain confidence level. There exists a mixed strategy Nash equilibrium of a chance-constrained game if the payoff vector of each player follows a multivariate elliptically symmetric distribution 29]. Such a Nash equilibrium can be computed by solving an equivalent mathematical program 31. The characterization of the set of Nash equilibria of a chanceconstrained game using the solution set of a variational inequality is given in 32. The games where the probability distributions of players' payoffs are partially known is studied using distributionally robust approach [30. The authors showed the existence of a mixed strategy Nash equilibrium for two different two-moments based uncertainty sets. For each uncertainty set, they proposed an equivalent mathematical program whose global maximizer gives a Nash equilibrium. There is a limited literature on zero-sum chance-constrained games, see for instance [3, 5, 6, 8,

Recently, the games with deterministic payoff functions and chanceconstrained strategy set of each player have been introduced [25, 33. In [33], the authors considered a two player zero-sum matrix game with individual chance constraints. For the case of elliptical distributions, they showed that a saddle point equilibrium of the game can be computed by solving a primal-dual pair of second order cone programs. In [25], the authors considered an $n$-player general sum game with joint chance constraints. The non-convexity of joint chance constraint is considered as a major difficulty for the existence of a Nash equilibrium. Peng et al. 25] proposed a new convex reformulation of the joint chance constraint when the row vectors defining random linear constraints are independent and follow multivariate normal distributions. Then, they showed that there exists a Nash equilibrium of a chance-constrained game (CCG). A 
brief summary of various chance-constrained game models studied by Singh et al. is given in Table 1 .

In this paper, we consider an $n$-player non-cooperative game with expected value payoff functions and chance-constrained strategy sets defined in a distributionally robust framework. The random constraint vectors are independent and their probability distributions are not completely known. The only available information of a probability distribution is that it belongs to some distributional uncertainty set. We consider various types of distributional uncertainty sets which are constructed using partially available information of the underlying probability distribution. The first uncertainty set is based on the density of the random parameters where the normal distribution is the reference distribution. The distance between the true density function and the normal density function is defined using $\phi$-divergence [17. The other uncertainty sets are moments based, they are constructed with the information of mean vectors, covariance matrices and a support of a probability distribution [4, 7, 21]. One of the moments based uncertainty sets is defined on a nonnegative support. For $\phi$-divergence uncertainty set, we show that a distributionally robust chance-constrained game (DRCCG) problem is equivalent to a CCG problem. Therefore, the existence of a Nash equilibrium in this case directly follows from the case of known probability distribution 25. For each type of moments based uncertainty set, we propose a new convex reformulation of a joint chance constraint using a logarithmic transformation. Using convex reformulation of the players' strategy sets, we show that there exists a Nash equilibrium of a DRCCG under mild conditions on payoff functions. As an application, we consider a Cournot competition on electricity market comprising of a set of generation nodes and distribution nodes. The firms produce electricity at generation nodes and transmit to distribution nodes for the consumer. The transmission over long distances creates power losses and the firms want to keep their power losses below a certain threshold. The power losses are defined using random variables whose distributions are partially known. We model the transmission constraints as distributionally robust joint chance constraints. We performed numerical experiments by considering two electricity firms where a Nash equilibrium of the game is computed using a best response algorithm.

The games considered in this paper are significantly different from the ones considered in 30. The players' payoff functions defined using distributionally robust individual chance constraints in [30] comes within the class of payoff functions considered in this paper. Unlike in [30, we consider the strategy set for each player defined by a distributionally robust joint chance constraint, and introduce two new moments based uncertainty sets, where one uncertainty set has a nonnegative support, and a density based uncertainty set.

The rest of the paper is organized as follows. Section 2 contains the definition of a DRCCG. Section 3 shows the existence of a Nash equilibrium of a DRCCG for different types of distributional uncertainty sets. Section 4 presents an application from an electricity market. We conclude the paper in Section 5 
Table 1: Existing chance-constrained game models

\begin{tabular}{|c|c|c|c|c|}
\hline No. & Model & Payoffs & $\begin{array}{l}\text { Strategy } \\
\text { sets }\end{array}$ & Main Result \\
\hline 1 & $\begin{array}{lr}n \text {-player } & \text { finite } \\
\text { strategic } & \text { games } \\
\text { considered in } 29\end{array}$ & $\begin{array}{l}\text { payoff functions } \\
\text { are defined using a } \\
\text { chance constraint }\end{array}$ & $\begin{array}{l}\text { Mixed } \\
\text { strategy } \\
\text { setup }\end{array}$ & $\begin{array}{l}\text { A Mixed strategy Nash } \\
\text { equilibrium exists for } \\
\text { elliptical distributions }\end{array}$ \\
\hline 2 & $\begin{array}{l}\text { 2-player bi-matrix } \\
\text { game considered in } \\
31\end{array}$ & $\begin{array}{l}\text { payoff functions } \\
\text { are defined using a } \\
\text { chance constraint }\end{array}$ & $\begin{array}{l}\text { Mixed } \\
\text { strategy } \\
\text { setup }\end{array}$ & $\begin{array}{l}\text { Equivalent mathemat- } \\
\text { ical program to com- } \\
\text { pute Nash equilibrium } \\
\text { for elliptical distribu- } \\
\text { tions }\end{array}$ \\
\hline 3 & $\begin{array}{lr}n \text {-player } & \text { finite } \\
\text { strategic } & \text { games } \\
\text { considered in } 30\end{array}$ & $\begin{array}{l}\text { payoff functions } \\
\text { are defined using } \\
\text { a distributionally } \\
\text { robust rance } \\
\text { constraint }\end{array}$ & $\begin{array}{l}\text { Mixed } \\
\text { strategy } \\
\text { setup }\end{array}$ & $\begin{array}{l}\text { A Mixed strategy Nash } \\
\text { equilibrium exists } \\
\text { and it can be com- } \\
\text { puted using equivalent } \\
\text { mathematical pro- } \\
\text { gram for two different } \\
\text { two-moments based } \\
\text { uncertainty sets }\end{array}$ \\
\hline 4 & $\begin{array}{l}n \text {-player continu- } \\
\text { ous strategy games } \\
\text { considered in } 32\end{array}$ & $\begin{array}{l}\text { payoff functions } \\
\text { are defined using a } \\
\text { chance constraint }\end{array}$ & $\begin{array}{l}\text { A general } \\
\text { convex and } \\
\text { compact } \\
\text { continuous } \\
\text { strategy } \\
\text { sets }\end{array}$ & $\begin{array}{l}\text { For elliptically dis- } \\
\text { tributed payoffs, } \\
\text { Nash equilibria and } \\
\text { generalized Nash equi- } \\
\text { libria exists and are } \\
\text { characterized using } \\
\text { the solution sets of } \\
\text { variational inequality }\end{array}$ \\
\hline 5 & $\begin{array}{l}\text { 2- player zero-sum } \\
\text { matrix game con- } \\
\text { sidered in } 33\end{array}$ & $\begin{array}{l}\text { payoff matrix is de- } \\
\text { terministic }\end{array}$ & $\begin{array}{l}\text { strategy } \\
\text { sets are de- } \\
\text { fined using } \\
\text { individual } \\
\text { chance } \\
\text { constraints }\end{array}$ & $\begin{array}{l}\text { Saddle point equilibria } \\
\text { are characterized using } \\
\text { a primal-dual pair of } \\
\text { second order cone pro- } \\
\text { grams when random } \\
\text { constraint vectors fol- } \\
\text { low elliptical distribu- } \\
\text { tion }\end{array}$ \\
\hline 6 & $\begin{array}{l}n \text {-player game con- } \\
\text { sidered in } 25\end{array}$ & $\begin{array}{lr}\text { payoff } & \text { functions } \\
\text { are deterministic } \\
\text { and satisfies stan- } \\
\text { dard } \quad \text { continuity } \\
\text { and } & \text { concavity } \\
\text { assumptions }\end{array}$ & $\begin{array}{l}\text { Strategy } \\
\text { sets are } \\
\text { defined us- } \\
\text { ing a joint } \\
\text { chance } \\
\text { constraint }\end{array}$ & $\begin{array}{l}\text { A Nash equilibrium } \\
\text { exists when random } \\
\text { constraint vectors are } \\
\text { independent and follow } \\
\text { multivariate normal } \\
\text { distributions }\end{array}$ \\
\hline
\end{tabular}

\section{The model}

We consider an $n$-player non-cooperative game defined by the following objects:

$-I=\{1,2, \cdots, n\}$ is the set of players.

- $X^{i} \subset \mathbb{R}_{++}^{m_{i}}$ denotes the set of all strategies of player $i$ which is a convex and compact set; $\mathbb{R}_{++}^{m_{i}}\left(\mathbb{R}_{+}^{m_{i}}\right)$ denotes the positive (nonnegative) orthant of $\mathbb{R}^{m_{i}}$. The product set $X=\prod_{i \in I} X^{i}$ is the set of vectors of strategies of all the players and $X^{-i}=\prod_{j=1 ; j \neq i}^{n} X^{j}$ is the set of vectors of strategies of all the players but player $i$. A vector $\left(y^{i}, x^{-i}\right)$ represents a strategy profile 
where $y^{i}$ is the strategy of player $i$ and the strategy of player $j, j \neq i$, is $x^{j}$.

- Let $\xi: \Omega \rightarrow \mathbb{R}^{d}$ be a random vector defined on a probability space $(\Omega, \mathcal{F}, \mathbb{P})$. For each $\omega \in \Omega$, let $v_{i}(x, \xi(\omega))$ represents a real valued payoff function of player $i$ which is defined on $\prod_{i \in I} \mathbb{R}_{++}^{m_{i}} \times \mathbb{R}^{d}$. We consider the case where the probability distribution $P_{i}$ of $\xi$ is only partially known. The only information we have of $P_{i}$ is that it belongs to a certain uncertainty set $\mathcal{P}_{i}$. We consider the worst-case scenario where the payoff function of player $i$, $i \in I$, is defined by

$$
u_{i}(x)=\inf _{P_{i} \in \mathcal{P}_{i}} \mathbb{E}_{P_{i}}\left[v_{i}(x, \xi)\right],
$$

where $\mathbb{E}_{P_{i}}$ denotes the expectation operator associated with probability distribution $P_{i}$.

We consider the case where the strategies of player $i$ are further restricted by the following stochastic linear constraints

$$
A^{i} x^{i} \leq b^{i},
$$

where $A^{i}=\left[A_{1}^{i}, A_{2}^{i}, \cdots, A_{K_{i}}^{i}\right]^{T}$ is a $K_{i} \times m_{i}$ random matrix defined on the probability space $(\Omega, \mathcal{F}, \mathbb{P})$, and $b^{i} \in \mathbb{R}^{K_{i}} ; T$ denotes the transposition. For each $k=1,2, \cdots, K_{i}, A_{k}^{i}$ is the $k^{\text {th }}$ row of $A^{i}$. We consider the case where the constraints of player $i$ given by (1) are jointly satisfied with at least a given probability level $\alpha_{i}$. Let $F^{i}$ denote the probability distribution of $A^{i}$. In many practical situations, the probability distribution $F^{i}$ is not completely known. Therefore, we consider the worst case scenario where constraints (1) are jointly satisfied with at least $\alpha_{i}$ probability for all possible distributions within a certain distributional uncertainty set $\mathcal{D}_{i}$. Then, the constraint (1) can be defined as a distributionally robust joint chance constraint given by

$$
\inf _{F^{i} \in \mathcal{D}_{i}} \mathbb{P}\left\{A^{i} x^{i} \leq b^{i}\right\} \geq \alpha_{i} .
$$

When $\mathcal{D}_{i}, i \in I$, contains only multivariate normal distribution, DRCCG reduces to a CCG considered in [25]. For an $\alpha_{i} \in[0,1]$, the feasible strategy set of player $i$ is defined by

$$
S_{\alpha_{i}}^{i}=\left\{x^{i} \in X^{i} \mid \inf _{F^{i} \in \mathcal{D}_{i}} \mathbb{P}\left\{A^{i} x^{i} \leq b^{i}\right\} \geq \alpha_{i}\right\}, i \in I .
$$

Using standard notations, we denote $S_{\alpha}=\prod_{i \in I} S_{\alpha_{i}}^{i}$ and $S_{\alpha_{-i}}^{-i}=\prod_{j \in I ; j \neq i} S_{\alpha_{j}}^{j}$. We assume that the set $S_{\alpha_{i}}^{i}$ is non-empty, and the uncertainty sets $\mathcal{D}_{i}, i \in I$, and the probability level vector $\alpha=\left(\alpha_{i}\right)_{i \in I}$ are known to all the players. Then, the above DRCCG is a non-cooperative game with complete information. For a given $\alpha$, a strategy profile $x^{*}$ is said to be a Nash equilibrium of a DRCCG if and only if for each $i \in I$,

$$
u_{i}\left(x^{i *}, x^{-i *}\right) \geq u_{i}\left(x^{i}, x^{-i *}\right), \forall x^{i} \in S_{\alpha_{i}}^{i} .
$$

For the rest of the paper we have the following assumption on the players' payoff functions [22]. 
Assumption 1 For each player $i, i \in I$, the following conditions hold.

1. $v_{i}\left(x^{i}, x^{-i}, \xi\right)$ is a concave function of $x^{i}$ for every $\left(x^{-i}, \xi\right) \in X^{-i} \times \mathbb{R}^{d}$.

2. $v_{i}(\cdot)$ is a continuous function.

3. $\mathbb{E}_{P_{i}}\left[v_{i}(x, \xi)\right]$ is finite valued for any $x \in X$ and $P_{i} \in \mathcal{P}_{i}$.

4. $\mathcal{P}_{i}$ is weakly compact.

\section{Existence of Nash equilibrium for distributionally robust chance-constrained games}

In this section, we consider the case where the rows of $A^{i}$ are independent and a probability distribution of $A^{i}$ is not completely known. We only know that it belongs to some uncertainty set which is constructed from the partially available information about the distribution. We consider five different types of uncertainty sets and for each case we show that there exists a Nash equilibrium of a DRCCG.

\subsection{Density based uncertainty set}

The uncertainty sets based on density function are often considered in the literature 17. Such uncertainty sets are constructed by considering a reference distribution from estimated available data. The decision makers believe that their estimated distribution may not be correct but it is not very far from the true distribution. We assume a normal distribution as a reference distribution. We denote the normal density function of row vector $A_{k}^{i}$ by $f_{k 0}^{i}$. Then, the estimated density function $f_{0}^{i}$ of $A^{i}$ is also normal, and it is given by the product of the density functions $\left(f_{k 0}^{i}\right)_{k=1}^{K_{i}}$. The distance between the estimated density $f_{0}^{i}$ and the true density $f^{i}$ is modeled by $\phi$-divergence, which is defined as

$$
D_{i}^{\phi}\left(f^{i} \| f_{0}^{i}\right)=\int_{\mathbb{R}^{K_{i} m_{i}}} \phi\left(\frac{f^{i}(y)}{f_{0}^{i}(y)}\right) f_{0}^{i}(y) d y, \quad \forall i \in I .
$$

The uncertainty set of player $i, i \in I$, is defined as

$$
\mathcal{D}_{i}^{\phi}=\left\{F^{i} \mid D_{i}^{\phi}\left(f^{i} \| f_{0}^{i}\right) \leq \varepsilon_{i}\right\}
$$

where $F^{i}$ is a probability distribution corresponding to the density function $f^{i}$ and $\varepsilon_{i}$ is divergence tolerance which represents the risk-aversion level of player $i$. Moreover, $\phi(s)$, called the $\phi$-divergence function, is a convex function on $s \geq$ 0 . It can be extended to $\mathbb{R}$ by setting $\phi(s)=+\infty$ for $s<0$. The $\phi$-divergence function takes value zero if $f^{i}(y)>0$ and $f_{0}^{i}(y)>0$ have the same value, i.e., $\phi(1)=0$. When the value of density function $f_{0}^{i}(y)$ is zero at some points, the terms $f_{0}^{i}(y) \phi\left(\frac{f^{i}(y)}{f_{0}^{i}(y)}\right)$ used in (3) are defined as $0 \phi\left(\frac{s}{0}\right):=s \lim _{p \rightarrow+\infty} \phi(p) / p$ for $s>0$, and $0 \phi\left(\frac{0}{0}\right):=0$. 
Let $\phi^{*}$ be a conjugate function of $\phi$ defined as $\phi^{*}(t)=\sup _{s \in \mathbb{R}}\{t y-\phi(s)\}$. Define, $\underline{m}\left(\phi^{*}\right)=\sup \left\{m \in R: \phi^{*}\right.$ is a finite constant on $\left.(-\infty, m]\right\}$ and $\bar{m}\left(\phi^{*}\right)=\inf \left\{m \in R: \phi^{*}(m)=+\infty\right\}$. Let $F_{0}^{i}$ be a probability distribution corresponding to density function $f_{0}^{i}$. Then, from Theorem 1 of [17] we have

$$
\inf _{F^{i} \sim \mathcal{D}_{i}^{\phi}} \mathbb{P}\left\{A^{i} x^{i} \leq b^{i}\right\} \geq \alpha_{i}=\mathbb{P}_{A^{i} \sim F_{0}^{i}}\left\{A^{i} x^{i} \leq b^{i}\right\} \geq \hat{\alpha}_{i},
$$

where $\hat{\alpha}_{i}=\min \left\{\alpha_{i}^{\prime}, 1\right\}$ such that

$$
\alpha_{i}^{\prime}=\inf _{z>0, \underline{m}\left(\phi^{*}\right) \leq z_{0}+z \leq \bar{m}\left(\phi^{*}\right)}\left\{\frac{\phi^{*}\left(z_{0}+z\right)-z_{0}-\left(1-\alpha_{i}\right) z+\varepsilon_{i}}{\phi^{*}\left(z_{0}+z\right)-\phi^{*}\left(z_{0}\right)}\right\} .
$$

In (4), $A^{i} \sim F_{0}^{i}$ denotes that $F_{0}^{i}$ is a probability distribution of $A^{i}$ and the probability on the right hand side is defined according to $F_{0}^{i}$.

The values of $\underline{m}\left(\phi^{*}\right)$ and $\bar{m}\left(\phi^{*}\right)$ for certain $\phi$-divergences are given in Table 1 of [17. We summarize the values of $\alpha_{i}^{\prime}$ for some famous $\phi$-divergences obtained from (5) in Table 2. For detailed proofs for these $\alpha_{i}^{\prime}$ we refer the readers to the Appendices of Jiang and Guan [17. By using (4), the strategy

Table 2: The values $\alpha_{i}^{\prime}$ for various $\phi$-divergences

\begin{tabular}{|c|c|c|c|}
\hline S.No. & $\phi(y), y \geq 0$ & $\alpha_{i}$ & $\alpha_{i}^{\prime}$ \\
\hline 1 & $(y-1)^{2}$ & $(0.5,1]$ & $\alpha_{i}+\frac{\sqrt{\varepsilon_{i}^{2}+4 \varepsilon_{i} \alpha_{i}\left(1-\alpha_{i}\right)}-\left(2 \alpha_{i}-1\right) \varepsilon_{i}}{2 \varepsilon_{i}+2}$ \\
\hline 2 & $|y-1|$ & {$[0,1]$} & $\alpha_{i}+\frac{\varepsilon_{i}}{2}$ \\
\hline 3 & $y \log y-y+1$ & {$[0,1]$} & $\inf _{y \in(0,1)}\left\{\frac{e^{-\varepsilon_{i}} y^{\alpha_{i}}-1}{y-1}\right\}$ \\
\hline
\end{tabular}

set $S_{\alpha_{i}}^{i}$ of player $i, i \in I$, can be written as

$$
S_{\alpha_{i}}^{i}=\left\{x^{i} \in X^{i} \mid \mathbb{P}_{A^{i} \sim F_{0}^{i}}\left\{A^{i} x^{i} \leq b^{i}\right\} \geq \hat{\alpha}_{i}\right\},
$$

where $\hat{\alpha}_{i}$ can be obtained using (5). Under Assumption 1, the payoff function $u_{i}\left(x^{i}, x^{-i}\right), i \in I$, is a concave function of $x^{i}$ for every $x^{-i}$. The strategy set $S_{\alpha_{i}}^{i}, i \in I$, defined by (6) has a convex and compact reformulation [25]. We further assume that $u_{i}\left(x^{i}, x^{-i}\right), i \in I$, is a non-increasing function of $x^{i}$ for every $x^{-i}$. Then, it follows from Theorem 3.14 of [25] that there exists a Nash equilibrium of a DRCCG.

\subsection{Moments based uncertainty set}

We often encounter practical situations where we only have some information about the first two moments of underlying probability distribution. We consider four different two-moments based uncertainty sets and for each uncertainty set, we propose a new convex reformulation of the distributionally robust joint chance constraint (2). Under the convex reformulation, we show that there exists a Nash equilibrium of a DRCCG. 
3.2.1 Uncertainty set with known first two order moments

We consider the uncertainty set of player $i, i \in I$, which accounts for the information about a mean vector $\mu_{k}^{i}$ and a covariance matrix $\Sigma_{k}^{i}$ of $\left(A_{k}^{i}\right)^{T}$ for all $k=1,2, \cdots, K_{i}$. For each $i \in I$, define

$\mathcal{D}_{i}^{k}\left(\mu_{k}^{i}, \Sigma_{k}^{i}\right)=\left\{\begin{array}{l|l}F_{k}^{i} & \begin{array}{l}\mathbb{E}_{F_{k}^{i}}\left[\left(A_{k}^{i}\right)^{T}\right]=\mu_{k}^{i}, \\ \mathbb{E}_{F_{k}^{i}}\left[\left(\left(A_{k}^{i}\right)^{T}-\mu_{k}^{i}\right)\left(\left(A_{k}^{i}\right)^{T}-\mu_{k}^{i}\right)^{T}\right]=\Sigma_{k}^{i}\end{array}\end{array}\right\}, k=1, \cdots, K_{i}$,

where $F_{k}^{i}$ is a probability distribution of $A_{k}^{i}$ and $\mathbb{E}_{F_{k}^{i}}$ is the expectation operator associated with $F_{k}^{i}$. Under independent assumption on the row vectors of matrix $A^{i}[9$, the distributionally robust joint chance constraint $(2)$ is satisfied if and only if there exists a vector $z^{i} \in \mathbb{R}^{K_{i}}$ such that $\sum_{k=1}^{K_{i}} z_{k}^{i}=1, z_{k}^{i} \geq 0$ for all $k=1,2, \cdots, K_{i}$ and

$$
\inf _{F_{k}^{i} \in \mathcal{D}_{i}^{k}\left(\mu_{k}^{i}, \Sigma_{k}^{i}\right)} \mathbb{P}\left\{A_{k}^{i} x^{i} \leq b_{k}^{i}\right\} \geq \alpha_{i}^{z_{k}^{i}}, \quad k=1,2, \cdots K_{i} .
$$

Then, using the deterministic reformulation of distributionally robust individual chance constraints (8) from [4, 12, we have the following deterministic reformulation for the distributionally robust joint chance constraint (2)

$$
Q_{\alpha_{i}}^{i}=\left\{\begin{array}{l}
(i)\left(\mu_{k}^{i}\right)^{T} x^{i}+\sqrt{\frac{\alpha_{i}^{z_{k}^{i}}}{1-\alpha_{i}^{z_{k}^{i}}}}\left\|\left(\Sigma_{k}^{i}\right)^{1 / 2} x^{i}\right\| \leq b_{k}^{i}, \forall k=1,2, \cdots, K_{i}, \\
(i i) \sum_{k=1}^{K_{i}} z_{k}^{i}=1, \\
(i i i) z_{k}^{i} \geq 0, \forall k=1,2, \cdots, K_{i},
\end{array}\right.
$$

where $\|\cdot\|$ is the Euclidean norm. It is clear that $Q_{\alpha_{i}}^{i}$ is not a convex set due to constraint $(i)$ of $(9)$. We reformulate the constraint $(i)$ of $(9)$ by using a change of variables technique under logarithmic transformation. We transform the vector $x^{i} \in X^{i}$ into a vector $y^{i} \in \mathbb{R}^{m_{i}}$, where $y_{j}^{i}=\ln x_{j}^{i}, j=1,2, \ldots, m_{i}$ [20. Under logarithmic transformation, we have the following reformulation of (9)

$$
\widetilde{Q}_{\alpha_{i}}^{i}=\left\{\begin{array}{l}
(i)\left(\mu_{k}^{i}\right)^{T} e^{y^{i}}+\left\|\left(\Sigma_{k}^{i}\right)^{1 / 2} e^{\frac{1}{2}\left(z_{k}^{i} \log \alpha_{i}-\log \left(1-\alpha_{i}^{z_{k}^{i}}\right)\right) \cdot \mathbb{1}_{m_{i}}+y^{i}}\right\| \leq b_{k}^{i}, \\
(i i) \sum_{k=1}^{K_{i}} z_{k}^{i}=1, \\
(i i i) z_{k}^{i} \geq 0, \forall k=1,2, \cdots, K_{i},
\end{array}\right.
$$


Let $Y^{i}$ be an image of $X^{i}$ under logarithmic transformation. Since, the logarithmic function is continuous and $X^{i}$ is a compact set, $Y^{i}$ is also a compact set. The convexity need not be preserved under logarithmic transformation. From now onward, we consider the set $X^{i}$ for which the set $Y^{i}$ remains convex. Such sets indeed exists, see for instance [25]. The reformulation of feasible strategy set $S_{\alpha_{i}}^{i}$ of player $i, i \in I$, is given by

$$
\widetilde{S}_{\alpha_{i}}^{i}=\left\{\left(y^{i}, z^{i}\right) \in Y^{i} \times \mathbb{R}^{K_{i}} \mid\left(y^{i}, z^{i}\right) \in \widetilde{Q}_{\alpha_{i}}^{i}\right\},
$$

and it is a compact set.

Assumption 2 For each $i \in I$ and $k=1,2, \ldots, K_{i}$, all the components of $\Sigma_{k}^{i}$ and $\mu_{k}^{i}$ are nonnegative.

Under Assumption 2, we show that the set $\widetilde{S}_{\alpha_{i}}^{i}$ is convex. It suffices to show that the constraint $(i)$ of 10 is convex.

Lemma 1 For each $i \in I$, the set $\widetilde{S}_{\alpha_{i}}^{i}$ is a convex set, under Assumption 2. for all $\alpha_{i} \in(0,1)$.

Proof Fix $\alpha_{i} \in(0,1)$. The function $\log (1-p)$ is non-increasing and concave in $p$, and $\alpha_{i}^{z_{k}^{i}}$ is a convex function of $z_{k}^{i}$. Therefore, the composition function $\log \left(1-\alpha_{i}^{z_{k}^{i}}\right)$ is a concave function of $z_{k}^{i}$. Then, $\left(e^{\frac{1}{2}\left(z_{k}^{i} \log \alpha_{i}-\log \left(1-\alpha_{i}^{z_{k}^{i}}\right)\right) \cdot \mathbb{1}_{m_{i}}+y^{i}}\right)$ is an $m_{i} \times 1$ vector whose components are nonnegative convex functions. Therefore, it follows from Proposition 2.1 of [14] that $\left\|\left(\Sigma_{k}^{i}\right)^{1 / 2} e^{\frac{1}{2}\left(z_{k}^{i} \log \alpha_{i}-\log \left(1-\alpha_{i}^{z_{k}^{i}}\right)\right) \cdot \mathbb{1}_{m_{i}}+y^{i}}\right\|$ is a convex function. The term $\left(\mu_{k}^{i}\right)^{T} e^{y^{i}}$ is a convex function because $\mu_{k}^{i}$ is a nonnegative vector. Hence, the constraints

$\left(\mu_{k}^{i}\right)^{T} e^{y^{i}}+\left\|\left(\Sigma_{k}^{i}\right)^{1 / 2} e^{\frac{1}{2}\left(z_{k}^{i} \log \alpha_{i}-\log \left(1-\alpha_{i}^{z_{k}^{i}}\right)\right) \cdot \mathbb{1}_{m_{i}}+y^{i}}\right\| \leq b_{k}^{i}, \forall k=1,2, \cdots, K_{i}$,

are convex. It is easy to see that the other constraints of $\widetilde{S}^{i}\left(\alpha_{i}\right)$ are convex. Therefore, $\widetilde{S}_{\alpha_{i}}^{i}, i \in I$ is a convex set.

\subsubsection{Uncertainty set with unknown second order moment}

In this section, we consider the uncertainty set of player $i, i \in I$, which accounts for the information about a mean vector $\mu_{k}^{i}$ and an upper bound $\Sigma_{k}^{i} \succ 0$ on covariance matrix of $\left(A_{k}^{i}\right)^{T}$ for all $k=1,2, \cdots, K_{i}$. For each $i \in I$, define

$\mathcal{D}_{i}^{k}\left(\mu_{k}^{i}, \Sigma_{k}^{i}\right)=\left\{\begin{array}{l|l}F_{k}^{i} & \begin{array}{l}\mathbb{E}_{F_{k}^{i}}\left[\left(A_{k}^{i}\right)^{T}\right]=\mu_{k}^{i}, \\ \mathbb{E}_{F_{k}^{i}}\left[\left(\left(A_{k}^{i}\right)^{T}-\mu_{k}^{i}\right)\left(\left(A_{k}^{i}\right)^{T}-\mu_{k}^{i}\right)^{T}\right] \preceq \Sigma_{k}^{i}\end{array}\end{array}\right\}, k=1,2, \cdots, K_{i}$. 
Under independent assumption on the row vectors of $A^{i}$, it follows from 7 ] that the deterministic reformulation of distributionally robust joint chance constraint (2) for uncertainty set (7) is the same as for the uncertainty set 12 . and it is given by (9). Therefore, under logarithmic transformation described in Section 3.2.1, a convex and compact reformulation of feasible strategy set $S_{\alpha_{i}}^{i}, i \in I$, is given by (11).

\subsubsection{Uncertainty set with unknown moments}

We consider the case where the mean vector of $\left(A_{k}^{i}\right)^{T}$ lies in an ellipsoid of size $\gamma_{k 1}^{i} \geq 0$ centered at $\mu_{k}^{i}$ and the covariance matrix of $\left(A_{k}^{i}\right)^{T}$ lies in a positive semi-definite cone defined with a linear matrix inequality. For each $i \in I$ and $k=1,2 \cdots, K_{i}$, let $\Sigma_{k}^{i} \succ 0, \gamma_{k 2}^{i}>0$. We define the uncertainty set of each player $i \in I$ as follows

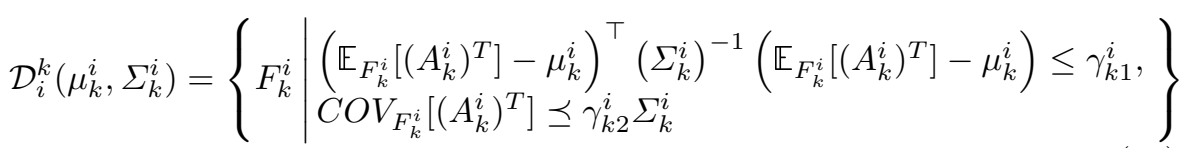

for all $k=1, \cdots, K_{i}$. $C O V_{F_{k}^{i}}$ is a covariance operator under probability distribution $F_{k}^{i}$. As mentioned earlier in Section 3.2.1 under independent assumption the chance constraint (2) can be equivalently written as

$$
\begin{gathered}
\inf _{F_{k}^{i} \in \mathcal{D}_{i}^{k}\left(\mu_{k}^{i}, \Sigma_{k}^{i}\right)} \mathbb{P}\left\{A_{k}^{i} x^{i} \leq b_{k}^{i}\right\} \geq \alpha_{i}^{z_{k}^{i}}, \\
\sum_{k=1}^{K_{i}} z_{k}^{i}=1, z_{k}^{i} \geq 0, k=1,2, \cdots, K_{i} .
\end{gathered}
$$

Based on the structure of the uncertainty set $\mathcal{D}_{i}^{k}\left(\mu_{k}^{i}, \Sigma_{k}^{i}\right)$, the constraint (14) can be written as

$$
\inf _{(\mu, \Sigma) \in \mathcal{U}_{k}^{i}} \inf _{F_{k}^{i} \in \mathcal{D}(\mu, \Sigma)} \mathbb{P}\left\{A_{k}^{i} x^{i} \leq b_{k}^{i}\right\} \geq \alpha_{i}^{z_{k}^{i}}
$$

where

$$
\mathcal{D}(\mu, \Sigma)=\left\{F_{k}^{i} \mid \mathbb{E}_{F_{k}^{i}}\left[\left(A_{k}^{i}\right)^{T}\right]=\mu, C O V_{F_{k}^{i}}\left[A_{k}^{i}\right]=\Sigma\right\}
$$

and

$$
\mathcal{U}_{k}^{i}=\left\{(\mu, \Sigma) \mid\left(\mu-\mu_{k}^{i}\right)^{\top}\left(\Sigma_{k}^{i}\right)^{-1}\left(\mu-\mu_{k}^{i}\right) \leq \gamma_{k 1}^{i}, \Sigma \preceq \gamma_{k 2}^{i} \Sigma_{k}^{i}\right\} .
$$

According to one-sided Chebyshev inequality [21, 27], we have

$$
\inf _{F_{k}^{i} \in \mathcal{D}(\mu, \Sigma)} \mathbb{P}\left\{A_{k}^{i} x^{i} \leq b_{k}^{i}\right\}= \begin{cases}1-\frac{1}{1+\frac{\left(\mu^{T} x^{i} b_{k}^{i}\right)^{2}}{\left(\left(x^{i}\right)^{T} \Sigma x^{2}\right)}}, & \text { if } \mu^{T} x^{i} \leq b_{k}^{i}, \\ 0, & \text { otherwise. }\end{cases}
$$


For the case $\mu^{T} x^{i}>b_{k}^{i}$,

$$
\inf _{F_{k}^{i} \in \mathcal{D}(\mu, \Sigma)} \mathbb{P}\left\{A_{k}^{i} x^{i} \leq b_{k}^{i}\right\}=0,
$$

and it leads constraint (14) to be infeasible. When $\mu^{T} x^{i} \leq b_{k}^{i}$, the constraint (14) is equivalent to

$$
\inf _{(\mu, \Sigma) \in \mathcal{U}_{k}^{i}} 1-\frac{1}{1+\left(\mu^{T} x^{i}-b_{k}^{i}\right)^{2} /\left(\left(x^{i}\right)^{T} \Sigma x^{i}\right)} \geq \alpha_{i}^{z_{k}^{i}},
$$

which can be reformulated as

$$
h_{k}^{i}\left(x^{i}\right) \geq \sqrt{\frac{\alpha_{i}^{z_{k}^{i}}}{1-\alpha_{i}^{z_{k}^{i}}}}
$$

where

$$
h_{k}^{i}\left(x^{i}\right)=\left\{\begin{array}{l}
\min _{\mu, \Sigma} \frac{b_{k}^{i}-\mu^{T} x^{i}}{\sqrt{\left(x^{i}\right)^{T} \Sigma x^{i}}} \\
\text { s.t. }(i)\left(\mu-\mu_{k}^{i}\right)^{\top}\left(\Sigma_{k}^{i}\right)^{-1}\left(\mu-\mu_{k}^{i}\right) \leq \gamma_{k 1}^{i}, \\
(i i) \Sigma \preceq \gamma_{k 2}^{i} \Sigma_{k}^{i} .
\end{array}\right.
$$

The problem 16 can be separated into two optimization problems. Therefore,

$$
h_{k}^{i}\left(x^{i}\right)=\frac{b_{k}^{i}+v_{1}\left(x^{i}\right)}{\sqrt{v_{2}\left(x^{i}\right)}}
$$

where

$$
\begin{gathered}
v_{1}\left(x^{i}\right)=\left\{\begin{array}{l}
\min _{\mu}-\mu^{T} x^{i} \\
\text { s.t. }\left(\mu-\mu_{k}^{i}\right)^{\top}\left(\Sigma_{k}^{i}\right)^{-1}\left(\mu-\mu_{k}^{i}\right) \leq \gamma_{k 1}^{i},
\end{array}\right. \\
v_{2}\left(x^{i}\right)=\left\{\begin{array}{l}
\max _{\Sigma}\left(x^{i}\right)^{T} \Sigma x^{i} \\
\text { s.t. } \Sigma \preceq \gamma_{k 2}^{i} \Sigma_{k}^{i} .
\end{array}\right.
\end{gathered}
$$

Let $\lambda \geq 0$ be a Lagrange multiplier associated with the constraint of optimization problem (17). By applying the KKT conditions, the optimal solution of 17) is given by $\mu=\mu_{k}^{i}+\frac{\sqrt{\gamma_{k 1}^{i}} \Sigma_{k}^{i} x^{i}}{\sqrt{\left(x^{i}\right)^{T} \sum_{k}^{i} x^{i}}}$ and associated Lagrange multiplier is given by $\lambda=\sqrt{\frac{\left(x^{i}\right)^{T} \sum_{k}^{i} x^{i}}{4 \gamma_{k 1}^{i}}}$. Therefore, the corresponding optimal value $v_{1}\left(x^{i}\right)=-\left(\mu_{k}^{i}\right)^{T} x^{i}-\sqrt{\gamma_{k 1}^{i}} \sqrt{\left(x^{i}\right)^{T} \Sigma_{k}^{i} x^{i}}$. Since, $u^{T} \Sigma u \leq u^{T} \gamma_{k 2}^{i} \Sigma_{k}^{i} u$ for any $u \in \mathbb{R}^{n}$, then, $v_{2}\left(x^{i}\right)=\gamma_{k 2}^{i}\left(x^{i}\right)^{T} \Sigma_{k}^{i} x^{i}$. Using this, constraint 15) can be reformulated as

$$
\left(\mu_{k}^{i}\right)^{T} x^{i}+\left(\sqrt{\frac{\alpha_{i}^{z_{k}^{i}}}{1-\alpha_{i}^{z_{k}^{i}}}} \sqrt{\gamma_{k 2}^{i}}+\sqrt{\gamma_{k 1}^{i}}\right)\left\|\left(\Sigma_{k}^{i}\right)^{1 / 2} x^{i}\right\| \leq b_{k}^{i} .
$$


Hence, we have the following deterministic reformulation for the distributionally robust joint chance constraint (2)

$$
Q_{\alpha_{i}}^{i}= \begin{cases}(i)\left(\mu_{k}^{i}\right)^{T} x^{i}+\left(\sqrt{\frac{\alpha_{i}^{z_{k}^{i}}}{1-\alpha_{i}^{z_{k}^{i}}}} \sqrt{\gamma_{k 2}^{i}}+\sqrt{\gamma_{k 1}^{i}}\right)\left\|\left(\Sigma_{k}^{i}\right)^{1 / 2} x^{i}\right\| \leq b_{k}^{i}, & \forall k=1,2, \cdots, K_{i}, \\
\text { (ii) } \sum_{k=1}^{K_{i}} z_{k}^{i}=1, \\
\left(\begin{array}{l}
\text { (iii) } z_{k}^{i} \geq 0, \forall k=1,2, \cdots, K_{i} .
\end{array}\right.\end{cases}
$$

For a convex reformulation of $(19)$, we use logarithmic transformation described in Section 3.2.1. The new convex reformulation of 19 is given by

$$
\widetilde{Q}_{\alpha_{i}}^{i}=\left\{\begin{aligned}
&(i)\left(\mu_{k}^{i}\right)^{T} e^{y^{i}}+\sqrt{\gamma_{k 2}^{i}} \|\left(\Sigma_{k}^{i}\right)^{1 / 2} e^{\frac{1}{2}\left(z_{k}^{i} \log \alpha_{i}-\log \left(1-\alpha_{i}^{z_{k}^{i}}\right)\right) \cdot \mathbb{1}_{m_{i}}+y^{i} \|} \\
&+\sqrt{\gamma_{k 1}^{i}}\left\|\left(\Sigma_{k}^{i}\right)^{1 / 2} e^{y^{i}}\right\| \leq b_{k}^{i}, \forall k=1,2, \cdots, K_{i}, \\
&(i i) \sum_{k=1}^{K_{i}} z_{k}^{i}=1, \\
&(i i i) z_{k}^{i} \geq 0, \forall k=1,2, \cdots, K_{i} .
\end{aligned}\right.
$$

Hence, the reformulation of feasible strategy set $S_{\alpha_{i}}^{i}$ of player $i, i \in I$, is given by

$$
\widetilde{S}_{\alpha_{i}}^{i}=\left\{\left(y^{i}, z^{i}\right) \in Y^{i} \times \mathbb{R}^{K_{i}} \mid\left(y^{i}, z^{i}\right) \in \widetilde{Q}_{\alpha_{i}}^{i}\right\} .
$$

The reformulation 20 is similar to 10$)$ except in constraint $(i)$ where there is one extra term and a positive multiplier in the second term. The convexity of (20) follows from the similar arguments used in Lemma 1. It is also a compact set. Therefore, the reformulated feasible strategy set $S_{\alpha_{i}}^{i}$ of player $i$ for distributional uncertainty set defined by $\sqrt{13}$ is a convex and compact set.

\subsubsection{Uncertainty set with known first order moment and nonnegative support}

So far we have considered the full support for the random vector $A_{k}^{i}$ in the uncertainty sets. However, in some cases the random vector $A_{k}^{i}$ can be nonnegative. To the best of our knowledge, distributionally robust games with nonnegative support have not been considered so far in the literature. To maintain the feasibility of the chance constraint (2), we further assume that $b_{k}^{i}>0$ for all $k=1,2, \cdots, K_{i}$. We define the uncertainty set for player $i, i \in I$, as follows

$$
\mathcal{D}_{i}^{k}\left(\mu_{k}^{i}\right)=\left\{F_{k}^{i} \mid \mathbb{E}_{F_{k}^{i}}\left[\left(A_{k}^{i}\right)^{T}\right]=\mu_{k}^{i}, \mathbb{P}_{F_{k}^{i}}\left[A_{k}^{i} \geq 0\right]=1\right\}, k=1, \ldots, K_{i},
$$


where $\mu_{k}^{i} \geq 0$ for all $k=1, \cdots, K_{i}$. Under independent assumption the chance constraint (2) can be equivalently written as

$$
\left.\begin{array}{l}
\inf _{F_{k}^{i} \in \mathcal{D}_{i}^{k}\left(\mu_{k}^{i}\right)} \mathbb{P}\left\{A_{k}^{i} x^{i} \leq b_{k}^{i}\right\} \geq z_{k}^{i}, k=1,2, \cdots, K_{i} \\
\prod_{k=1}^{K_{i}} z_{k}^{i} \geq \alpha_{i}, 0 \leq z_{k}^{i} \leq 1, k=1,2, \cdots, K_{i} .
\end{array}\right\}
$$

For each $k=1,2, \cdots, K_{i}$, consider the optimization problem $\inf _{F_{k}^{i} \in \mathcal{D}_{i}^{k}\left(\mu_{k}^{i}\right)} \mathbb{P}\left\{A_{k}^{i} x^{i} \leq b_{k}^{i}\right\}$ which can be reformulated as

$$
\begin{aligned}
& \inf _{F_{k}^{i}} \int_{A_{k}^{i} \geq 0} \mathbf{1}_{A_{k}^{i} x^{i} \leq b_{k}^{i}} \mathrm{~d} F_{k}^{i}\left(A_{k}^{i}\right) \\
& \text { s.t. } \\
& \int_{A_{k}^{i} \geq 0}\left(A_{k}^{i}\right)^{T} \mathrm{~d} F_{k}^{i}\left(A_{k}^{i}\right)=\mu_{k}^{i} \\
& \qquad \int_{A_{k}^{i} \geq 0} \mathrm{~d} F_{k}^{i}\left(A_{k}^{i}\right)=1
\end{aligned}
$$

where $\mathbf{1}_{\mathbf{C}}$ is the indicator function over a given set $\mathbf{C}$. The dual problem of (23) is given by

$$
\begin{array}{cl}
\sup _{\beta_{k}^{i} \in \mathbb{R}^{m_{i}}, \lambda_{k}^{i} \in \mathbb{R}}\left(\mu_{k}^{i}\right)^{T} \beta_{k}^{i}+\lambda_{k}^{i} \\
\text { s.t. } & \mathbf{1}_{A_{k}^{i} x^{i} \leq b_{k}^{i}}-A_{k}^{i} \beta_{k}^{i}-\lambda_{k}^{i} \geq 0, \forall A_{k}^{i} \geq 0 .
\end{array}
$$

The strong duality follows from 28] because Dirac measure $\delta_{\mu_{k}^{i}}$ lies in the relative interior of $\mathcal{D}_{i}^{k}\left(\mu_{k}^{i}\right)$. The constraint 24) can be reformulated as

$$
\begin{aligned}
& A_{k}^{i} \beta_{k}^{i}+\lambda_{k}^{i} \leq 1, \forall A_{k}^{i} \geq 0, \\
& A_{k}^{i} \beta_{k}^{i}+\lambda_{k}^{i} \leq 0, \forall A_{k}^{i} \geq 0, A_{k}^{i} x^{i}>b_{k}^{i} .
\end{aligned}
$$

Constraint (25) is equivalent to $\beta_{k}^{i} \leq 0$ and $\lambda_{k}^{i} \leq 1$. Since, we look for the supremum in (24), we can replace $A_{k}^{i} x^{i}>b_{k}^{i}$ in (26) by $A_{k}^{i} x^{i} \geq b_{k}^{i}$. Then, using duality theory of linear programming, the constraint 26) can be equivalently written as

$$
\begin{gathered}
\lambda_{k}^{i} \leq b_{k}^{i} \tau_{k}^{i}, \tau_{k}^{i} \geq 0 \\
\beta_{k}^{i}+\tau_{k}^{i} x^{i} \leq 0
\end{gathered}
$$

Since, there is no duality gap between (23) and (24), using (22) we have the following deterministic reformulation for the distributionally robust joint chance 
constraint (2)

$$
Q_{\alpha_{i}}^{i}=\left\{\begin{array}{l}
(i)\left(\mu_{k}^{i}\right)^{T} \beta_{k}^{i}+\lambda_{k}^{i} \geq z_{k}^{i}, k=1,2, \cdots, K_{i}, \\
(i i) \beta_{k}^{i} \leq 0, \lambda_{k}^{i} \leq 1, k=1,2, \cdots, K_{i}, \\
(i i i) \lambda_{k}^{i} \leq b_{k}^{i} \tau_{k}^{i}, \tau_{k}^{i} \geq 0, k=1,2, \cdots, K_{i}, \\
(i v) \beta_{k}^{i}+\tau_{k}^{i} x^{i} \leq 0, k=1,2, \cdots, K_{i}, \\
(v) \prod_{k=1}^{K_{i}} z_{k}^{i} \geq \alpha_{i}, 0 \leq z_{k}^{i} \leq 1, k=1,2, \cdots, K_{i}
\end{array}\right.
$$

Since $\mu_{k}^{i} \geq 0, \beta_{k}^{i} \leq 0$ and $0 \leq z_{k}^{i} \leq 1$, constraint (i) in 27) implies $\lambda_{k}^{i} \geq 0$. In fact, it is easy to see that the components of vector $\left(\tau_{k}^{i}, \lambda_{k}^{i}, z_{k}^{i},-\beta_{k}^{i}\right)$ are positive. Therefore, we use change of variables under logarithmic transformation as follows: $\tilde{\lambda}_{k}^{i}=\log \left(\lambda_{k}^{i}\right), \tilde{\beta}_{k j}^{i}=\log \left(-\beta_{k j}^{i}\right), \tilde{\tau}_{k}^{i}=\log \left(\tau_{k}^{i}\right), \zeta_{k}^{i}=\log \left(z_{k}^{i}\right)$, and $y_{j}^{i}=\ln x_{j}^{i}$, for all $j=1,2, \ldots, m_{i}, k=1,2, \ldots, K_{i}$. We have a new convex reformulation of (27) given by

$$
\widetilde{Q}_{\alpha_{i}}^{i}=\left\{\begin{array}{l}
(i) e^{\zeta_{k}^{i}-\tilde{\lambda_{k}^{i}}}+\left(\mu_{k}^{i}\right)^{T} e^{\tilde{\beta_{k}^{i}}-\tilde{\lambda}_{k}^{i} \mathbf{1}_{m_{i}}} \leq 1, k=1,2, \cdots, K_{i}, \\
(i i) \tilde{\lambda_{k}^{i}} \leq 0, k=1,2, \cdots, K_{i}, \\
(i i i) \tilde{\lambda_{k}^{i}} \leq \tilde{\tau_{k}^{i}}+\log \left(b_{k}^{i}\right), k=1,2, \cdots, K_{i}, \\
(i v) \tilde{\tau_{k}^{i}}+y_{j}^{i}-\beta_{k j}^{i} \leq 0, k=1,2, \cdots, K_{i}, j=1, \cdots, m_{i}, \\
(v) \sum_{k=1}^{K_{i}} \zeta_{k}^{i} \geq \log \left(\alpha_{i}\right), \zeta_{k}^{i} \leq 0, k=1,2, \cdots, K_{i}
\end{array}\right.
$$

Hence, the reformulation of feasible strategy set $S_{\alpha_{i}}^{i}$ of player $i, i \in I$, is given by

$$
\widetilde{S}_{\alpha_{i}}^{i}=\left\{\left(y^{i}, \tilde{\lambda}^{i}, \tilde{\beta}^{i}, \tilde{\tau}^{i}, \zeta^{i}\right) \in Y^{i} \times \mathbb{R}^{K_{i}} \times \mathbb{R}^{K_{i} \cdot m_{i}} \times \mathbb{R}^{K_{i}} \times \mathbb{R}^{K_{i}} \mid\left(y^{i}, \tilde{\lambda}^{i}, \tilde{\beta}^{i}, \tilde{\tau}^{i}, \zeta^{i}\right) \in \widetilde{Q}_{\alpha_{i}}^{i}\right\} .
$$

It is evident that $\widetilde{S}_{\alpha_{i}}^{i}$ is a convex set. It follows from (27) that $Q_{\alpha_{i}}^{i}$ is a compact set. Then, under logarithmic transformation $\widetilde{S}_{\alpha_{i}}^{i}, i \in I$, is a compact set.

We present a general theorem on the existence of a Nash equilibrium for a DRCCG which covers the case of each uncertainty set as a special case. The concavity of a player's payoff function in its strategies, for a fixed strategy profile of other players, plays a crucial role in showing the existence of a Nash equilibrium. Therefore, we need the following assumption on players' payoff functions which ensures the required concavity property of the payoff functions under logarithmic transformation.

Assumption 3 For each player $i, i \in I$, the payoff function $u_{i}\left(\cdot, x^{-i}\right)$ satisfies the non-increasing condition for every $x^{-i} \in X^{-i}$, i.e., for any two points $x^{i}$ and $\bar{x}^{i}$ such that $x_{k}^{i} \leq \bar{x}_{k}^{i}$ for all $k=1,2, \ldots, m_{i}$, we have $u_{i}\left(x^{i}, x^{-i}\right) \geq$ $u_{i}\left(\bar{x}^{i}, x^{-i}\right)$. 
Theorem 1 Consider an n-player DRCCG, where

1. the payoff function of player $i, i \in I$, satisfies the Assumptions 1 and 3 ,

2. the reformulation of feasible strategy set $S_{\alpha_{i}}^{i}, i \in I$, under logarithmic transformation is a convex and compact set.

Then, there exists a Nash equilibrium of a DRCCG for all $\alpha \in(0,1)^{n}$.

Proof Let $\alpha \in(0,1)^{n}$. Under Assumption 1, the payoff function $u_{i}\left(x^{i}, x^{-i}\right)$, $i \in I$, is a concave function of $x^{i}$ for every $x^{-i} \in X^{-i}$, and a continuous function of $x$. For each $i \in I$, define a composition function $V_{i}=u_{i} \circ d_{i}$, where $d_{i}: \mathbb{R}^{m_{1}} \times \mathbb{R}^{m_{2}} \times \cdots \times \mathbb{R}^{m_{n}} \rightarrow \mathbb{R}_{++}^{m_{1}} \times \mathbb{R}_{++}^{m_{2}} \times \cdots \times \mathbb{R}_{++}^{m_{n}}$, such that

$$
d_{i}\left(y^{1}, y^{2}, \cdots, y^{n}\right)=\left(e^{y^{1}}, e^{y^{2}}, \cdots, e^{y^{n}}\right) .
$$

Under Assumptions 3, the composition function $V_{i}\left(\cdot, y^{-i}\right), i \in I$, is a concave function of $y^{i}$ for every $y^{-i}$ and $V_{i}(\cdot)$ is a continuous function of $y$. Let $\widetilde{S}_{\alpha_{i}}^{i}$, $i \in I$, be the reformulation of the feasible strategy set $S_{\alpha_{i}}^{i}$ under logarithmic transformation which is a convex and compact set. Then, there exists a Nash equilibrium of an $n$-player non-cooperative game defined by strategy sets $\left(\widetilde{S}_{\alpha_{i}}^{i}\right)_{i \in I}$ and payoff functions $\left(V_{i}(\cdot)\right)_{i \in I}$ [11, 13]. Therefore, there exists $y^{*} \in \prod_{i \in I} \widetilde{S}_{\alpha_{i}}^{i}$ such that

$$
V_{i}\left(y^{i *}, y^{-i *}\right) \geq V_{i}\left(y^{i}, y^{-i *}\right), \forall y^{i} \in \widetilde{S}_{\alpha_{i}}^{i}
$$

Under the hypothesis of the theorem, $\widetilde{S}_{\alpha_{i}}^{i}$ is a reformulation of $S_{\alpha_{i}}^{i}$, where $x^{i}=e^{y^{i}}$. Therefore, for $x^{*}=e^{y^{*}}$ we have

$$
u_{i}\left(x^{i *}, x^{-i *}\right) \geq u_{i}\left(x^{i}, x^{-i *}\right), \forall x^{i} \in S_{\alpha_{i}}^{i} .
$$

Hence, $x^{*}$ is a Nash equilibrium of a DRCCG for all $\alpha \in(0,1)^{n}$.

The reformulated feasible strategy sets $\widetilde{S}_{\alpha_{i}}^{i}, i \in I$, is a convex and compact set for each uncertainty set. Therefore, the corollary given below follows directly from Theorem 1 .

Corollary 1 Consider an n-player DRCCG, where

1. the payoff function of player $i, i \in I$, satisfies the Assumptions 1 and 3 .

2. the row vectors of $A^{i}, i \in I$ are independent,

3. one of the following conditions hold true

- for each $k=1,2, \cdots K_{i}$, the probability distribution of row vector $A_{k}^{i}$ belongs to uncertainty set $\mathcal{D}_{i}^{k}\left(\mu_{k}^{i}, \Sigma_{k}^{i}\right)$ defined as in (7), where the mean vector $\mu_{k}^{i}$ and the covariance matrix $\Sigma_{k}^{i}$ satisfy Assumption 2 . or

- for each $k=1,2, \cdots K_{i}$, the probability distribution of row vector $A_{k}^{i}$ belongs to uncertainty set $\mathcal{D}_{i}^{k}\left(\mu_{k}^{i}, \Sigma_{k}^{i}\right)$ defined as in 12 , where mean vector $\mu_{k}^{i}$ and the upper bound $\Sigma_{k}^{i}$ on covariance matrix satisfy Assumption 2.

or 
- for each $k=1,2, \cdots K_{i}$, the probability distribution of row vector $A_{k}^{i}$ belongs to uncertainty set $\mathcal{D}_{i}^{k}\left(\mu_{k}^{i}, \Sigma_{k}^{i}\right)$ defined as in (13), where $\mu_{k}^{i}$ and $\Sigma_{k}^{i}$ satisfy Assumption 2

or

- for each $k=1,2, \cdots K_{i}$, the probability distribution of row vector $A_{k}^{i}$ belongs to uncertainty set $\mathcal{D}_{i}^{k}\left(\mu_{k}^{i}\right)$ defined as in (21).

Then, there exists a Nash equilibrium of a DRCCG for all $\alpha \in(0,1)^{n}$.

\section{Cournot competition in electricity market}

We consider an electricity market where the firms compete over an electricity network comprises of a set of nodes. There are several generation nodes where the firms installed their generation facilities to produce electricity. There are also some distribution nodes from where the electricity is distributed to the consumers. The firms generate the electricity at their facilities and transmit to the distribution nodes. The transmission over long distances creates power losses which is better modeled using random variables. For simplicity, we omit the problem of consumers, which means any quantity of electricity can be consumed. The components of electricity market are described as follows:

\section{$I$ - set of firms called as players}

$N$ - the set of generation nodes

$N_{i}$ - subset of generation nodes where firm $i$ has installed its generation facilities $I_{k}$ - the set of firms who owns generation facilities at node $k$

$M-$ the set of distribution nodes

Let $x_{k}^{i}=\left(x_{k j}^{i}\right)_{j \in M}$ be a vector of quantities being transmitted from the generation node $k$ to the distribution nodes by firm $i$, e.g., $x_{k j}^{i}$ is the quantity transmitted from the generation node $k$ to the distribution node $j$ by firm $i$. Denote a generation level vector of firm $i$ by $x^{i}=\left(x_{k}^{i}\right)_{k \in N_{i}}$. We assume that the price at a generation node $k$ is different for each distribution node $j$ and it depends on the total amount of electricity being transmitted to the node $j$ from the node $k$. The price for a distribution node $j$ at a generation node $k$ is given by,

$$
P_{k j}=\beta_{k j}-\delta_{k j} \sum_{i \in I_{k}} x_{k j}^{i}, k \in N, j \in M,
$$

where $\delta_{k j} \geq 0$ for all $k \in N, j \in M$. Each firm incurs cost in generation as well as in the transmission of electricity. Let $c_{k j}^{i}\left(x_{k j}^{i}\right)$ be the cost incurred by firm $i$ for the generation and transmission of $x_{k j}^{i}$ unit of electricity from node $k$ to node $j$. We consider the linear cost function for each firm, i.e., $c_{k j}^{i}\left(x_{k j}^{i}\right)=c_{k j}^{i} x_{k j}^{i}$ for all $k \in N_{i}, j \in M, i \in I$. The payoff function of firm $i$ is 
given by

$$
u_{i}\left(x^{i}, x^{-i}\right)=\sum_{k \in N_{i}} \sum_{j \in M}\left(x_{k j}^{i}\left(\beta_{k j}-\delta_{k j} \sum_{l \in I_{k}} x_{k j}^{l}\right)-c_{k j}^{i} x_{k j}^{i}\right) .
$$

The case where parameters $\beta_{k j}, \delta_{k j}$ and $c_{k j}^{i}$ are random variables, e.g., when the market has randomness in the price and cost functions. In such cases, we take the average values of these parameters. The strategy set of player $i$ is defined as $X^{i}=\left\{x^{i}=\left(x_{k}^{i}\right)_{k \in N_{i}} \mid x_{k j}^{i} \in\left[\varepsilon_{k j}^{i}, \gamma_{k j}^{i}\right], k \in N_{i}, j \in M\right\}$, where $\varepsilon_{k j}^{i}>0$ and $\gamma_{k j}^{i}$ denote the minimum and maximum output of firm $i$ from node $k$ to node $j$, respectively. Let $a_{k}^{i}=\left(a_{k j}^{i}\right)_{j \in M}$ be a vector of unit electricity loss for firm $i$ at generation node $k$ where $a_{k j}^{i}$ represents the unit electricity loss during the transmission from the $k$ th generation node to the $j$ th distribution node. We assume that $a_{k j}^{i}$ is a random variable for each $i \in I, j \in M$ and $k \in N_{i}$. Each firm wants to keep its loss under a certain threshold. Let $b^{i}$ denotes the threshold vector for firm $i$. For a given confidence level $\alpha_{i}$, the distributionally robust joint chance constraint of firm $i, i \in I$, is given by

$$
\inf _{F^{i} \in \mathcal{D}_{i}} \mathbb{P}\left\{\left(a_{k}^{i}\right)^{T} x_{k}^{i} \leq b_{k}^{i}, k \in N_{i}\right\} \geq \alpha_{i} .
$$

We assume that the electricity loss for each firm at different node is independent, i.e., the random vectors $\left(a_{k}^{i}\right)_{k \in N_{i}}$ are independent. Then, under the logarithmic transformation, the payoff function of player $i, i=1,2$, is given by

$$
V_{i}\left(y^{i}, y^{-i}\right)=\sum_{k \in N_{i}} \sum_{j \in M}\left(e^{y_{k j}^{i}}\left(\beta_{k j}-\delta_{k j} \sum_{l \in I_{k}} e^{y_{k j}^{l}}\right)-c_{k j}^{i} e^{y_{k j}^{i}}\right) .
$$

For a fixed $y^{-i}$, the function $V_{i}\left(y^{i}, y^{-i}\right)$ is a concave function of $y^{i}$ if its Hessian matrix is negative semidefinite. The off-diagonal entries of the Hessian matrix are zero and the diagonal entries are given by

$$
\frac{\partial^{2} V_{i}\left(y^{i}, y^{-i}\right)}{\left(\partial y_{k j}^{i}\right)^{2}}=\left[\left(\beta_{k j}-\delta_{k j} \sum_{l \in I_{k}} e^{y_{k j}^{l}}-c_{k j}^{i}\right) \times e^{y_{k j}^{i}}\right]-\left[3 \times e^{2 y_{k j}^{i}} \times \delta_{k j}\right] \text {. }
$$

Then, the Hessian matrix is negative semidefinite if

$$
3 e^{y_{k j}^{i}}+\sum_{l \in I_{k}} e^{y_{k j}^{l}} \geq \frac{\beta_{k j}-c_{k j}^{i}}{\delta_{k j}}, k \in N_{i} j \in M .
$$

The condition 32 holds for the suitable choice of a minimum output, $\varepsilon_{k j}^{i}$, of the firms . The strategy set, under logarithmic transformation, is given by

$$
Y^{i}=\left\{y^{i}=\left(y_{k j}^{i}\right)_{k \in N_{i}, j \in M} \mid y_{k j}^{i} \in\left[\ln \varepsilon_{k j}^{i}, \ln \gamma_{k j}^{i}\right], k \in N_{i}, j \in M\right\} .
$$

It is clear that $Y^{i}$ is a convex and compact set. We consider the case of known mean $\mu_{k}^{i}$ and known co-variance matrix $\Sigma_{k}^{i}$ of $a_{k}^{i}$. In this case, the reformulated feasible strategy set $\tilde{S}_{\alpha_{i}}^{i}$ is given by (11). 
4.1 Case Study

For illustration purpose, we consider the case of two electricity firms whose generation nodes are the same. It is easy to see that the condition $(32)$ holds if

$$
\varepsilon_{k j}^{1} \geq \frac{3 \beta_{k j}-4 c_{k j}^{1}+c_{k j}^{2}}{15 \delta_{k j}}, \varepsilon_{k j}^{2} \geq \frac{3 \beta_{k j}-4 c_{k j}^{2}+c_{k j}^{1}}{15 \delta_{k j}}, k \in N, j \in M .
$$

Therefore, it follows from Corollary 1 that there exists a Nash equilibrium of the game. For a fixed $\left(y^{2}, z^{2}\right) \in \widetilde{S}_{\alpha_{2}}^{2}$, player 1 solves the following convex optimization problem

$$
\begin{aligned}
& {\left[P_{1}\right] \max _{y^{1}, z^{1}} V_{1}\left(y^{1}, y^{2}\right)} \\
& \text { s.t. }\left(y^{1}, z^{1}\right) \in \widetilde{S}_{\alpha_{1}}^{1} .
\end{aligned}
$$

The set of optimal solution of $\left[P_{1}\right]$, which is also called the best response set of player 1 , is given by

$$
B R_{1}\left(y^{2}\right)=\left\{\left(\bar{y}^{1}, \bar{z}^{1}\right) \mid V_{1}\left(\bar{y}^{1}, y^{2}\right) \geq V_{1}\left(y^{1}, y^{2}\right), \forall\left(y^{1}, z^{1}\right) \in \widetilde{S}_{\alpha_{1}}^{1}\right\}
$$

Similarly, for a fixed $\left(y^{1}, z^{1}\right) \in \tilde{S}_{\alpha_{1}}^{1}$, player 2 solves the following convex optimization problem

$$
\begin{aligned}
& {\left[P_{2}\right] \max _{y^{2}, z^{2}} V_{2}\left(y^{1}, y^{2}\right)} \\
& \text { s.t. }\left(y^{2}, z^{2}\right) \in \widetilde{S}_{\alpha_{2}}^{2} .
\end{aligned}
$$

The best response set of player 2 , is given by

$$
B R_{2}\left(y^{1}\right)=\left\{\left(\bar{y}^{2}, \bar{z}^{2}\right) \mid V_{2}\left(y^{1}, \bar{y}^{2}\right) \geq V_{2}\left(y^{1}, y^{2}\right), \forall\left(y^{2}, z^{2}\right) \in \widetilde{S}_{\alpha_{2}}^{2}\right\}
$$

It is clear that, if $\left(y^{1 *}, z^{1 *}\right) \in B R_{1}\left(y^{2 *}\right)$ and $\left(y^{2 *}, z^{2 *}\right) \in B R_{2}\left(y^{1 *}\right),\left(x^{1 *}, x^{2 *}\right)=$ $\left(e^{y^{1 *}}, e^{y^{2 *}}\right)$ is a Nash equilibrium of the game. For computational purpose, we use the best response algorithm as outlined below:

\section{Algorithm 1 (Best response algorithm) Step-1 Select initial feasi-} ble point $\left(y^{2(0)}, z^{2(0)}\right) \in \widetilde{S}_{\alpha_{2}}^{2}$ for player 2. Set $k:=0$.

Step-2 Solve convex optimization problem $\left[P_{1}\right]$ and find a point $\left(y^{1(k)}, z^{1(k)}\right) \in B R_{1}\left(y^{2(k)}\right)$.

Step-3 If $\left(y^{2(k)}, z^{2(k)}\right) \in B R_{1}\left(y^{1(k)}\right)$, then set $\left(x^{1 *}, x^{2 *}\right)=\left(e^{y^{1(k)}}, e^{y^{2(k)}}\right)$ and stop. Otherwise, solve convex optimization problem $\left[P_{2}\right]$ and find a point $\left(y^{2(k)}, z^{2(k)}\right) \in B R_{1}\left(y^{1(k)}\right)$, set $k=k+1$ and go to step 2 .

If the Algorithm 1 stops, $\left(x^{1 *}, x^{2 *}\right)$ is a Nash equilibrium of the game. The proof that Algorithm 1 never cycles is still an open problem. 
Example 1 We consider the case of two electricity firms with four generation nodes and three distribution nodes, i.e., $N=\{1,2,3,4\}$ and $M=\{1,2,3\}$. We take the confidence level values of player 1 and player 2 as 0.9 , i.e., $\alpha_{1}=$ $\alpha_{2}=0.9$. The randomly generated data used in the model are summarized as follows:

$$
\begin{aligned}
& \beta_{k j}=30, \delta_{k j}=1, c_{k j}^{1}=15, c_{k j}^{2}=12, \\
& \varepsilon_{k j}^{1}=3, \varepsilon_{k j}^{2}=4, \gamma_{k j}^{1}=\gamma_{k j}^{2}=50 \forall k \in N, j \in M, \\
& \mu_{1}^{1}=\left(\begin{array}{l}
0.237 \\
0.224 \\
0.240
\end{array}\right), \mu_{2}^{1}=\left(\begin{array}{l}
0.241 \\
0.226 \\
0.166
\end{array}\right), \mu_{3}^{1}=\left(\begin{array}{c}
0.159 \\
0.239 \\
0.224
\end{array}\right), \mu_{4}^{1}=\left(\begin{array}{c}
0.167 \\
0.165 \\
0.186
\end{array}\right) \text {, } \\
& \mu_{1}^{2}=\left(\begin{array}{l}
0.214 \\
0.182 \\
0.246
\end{array}\right), \mu_{2}^{2}=\left(\begin{array}{c}
0.218 \\
0.155 \\
0.194
\end{array}\right), \mu_{3}^{2}=\left(\begin{array}{c}
0.197 \\
0.162 \\
0.211
\end{array}\right), \mu_{4}^{2}=\left(\begin{array}{c}
0.212 \\
0.195 \\
0.171
\end{array}\right) \text {, } \\
& \Sigma_{1}^{1}=\left(\begin{array}{lll}
0.074 & 0.056 & 0.034 \\
0.056 & 0.058 & 0.022 \\
0.034 & 0.022 & 0.035
\end{array}\right), \Sigma_{2}^{1}=\left(\begin{array}{lll}
0.024 & 0.007 & 0.017 \\
0.007 & 0.009 & 0.006 \\
0.017 & 0.006 & 0.021
\end{array}\right), \\
& \Sigma_{3}^{1}=\left(\begin{array}{ccc}
0.01 & 0.016 & 0.010 \\
0.016 & 0.065 & 0.038 \\
0.010 & 0.038 & 0.037
\end{array}\right), \Sigma_{4}^{1}=\left(\begin{array}{lll}
0.042 & 0.035 & 0.040 \\
0.035 & 0.070 & 0.046 \\
0.040 & 0.046 & 0.058
\end{array}\right), \\
& \Sigma_{1}^{2}=\left(\begin{array}{lll}
0.043 & 0.026 & 0.030 \\
0.026 & 0.038 & 0.025 \\
0.030 & 0.025 & 0.036
\end{array}\right), \Sigma_{2}^{2}=\left(\begin{array}{lll}
0.045 & 0.037 & 0.022 \\
0.037 & 0.052 & 0.014 \\
0.022 & 0.014 & 0.023
\end{array}\right), \\
& \Sigma_{3}^{2}=\left(\begin{array}{lll}
0.048 & 0.029 & 0.039 \\
0.029 & 0.026 & 0.027 \\
0.039 & 0.027 & 0.044
\end{array}\right), \Sigma_{4}^{2}=\left(\begin{array}{lll}
0.006 & 0.002 & 0.003 \\
0.002 & 0.015 & 0.014 \\
0.003 & 0.014 & 0.034
\end{array}\right), \\
& b^{1}=\left(\begin{array}{c}
22.138 \\
21.519 \\
20.323 \\
20.921
\end{array}\right), b^{2}=\left(\begin{array}{c}
23.277 \\
21.393 \\
22.65 \\
24.968
\end{array}\right) \text {. }
\end{aligned}
$$

We implement Algorithm 1 on Intel@ Core $^{\text {TM }}$ 64-bit i5-7200U CPU @ $2.50 \mathrm{GHz} \times 4$ and $11.6 \mathrm{GiB}$ RAM on Ubuntu 18.04.2 LTS. We use sqp in GNU Octave 5.2.0 to solve the best response convex optimization problem of both the players. For the above data sets, Algorithm 1 converges to a point $\left(y^{1 *}, z^{1 *}, y^{2 *}, z^{2 *}\right)$ such that $\left(y^{1 *}, z^{1 *}\right) \in B R_{1}\left(y^{2 *}\right)$ and $\left(y^{2 *}, z^{2 *}\right) \in B R_{2}\left(y^{1 *}\right)$. Therefore, $\left(x^{1 *}, x^{2 *}\right)=\left(e^{y^{1 *}}, e^{y^{2 *}}\right)$ is a Nash equilibrium of the game and it is given by

$$
\begin{gathered}
x^{1 *}=((4.34,4.3,4.32),(4.4,4.38,4.25),(4.39,4.28,4.38),(4.04,4.10,4.14)), \\
x^{2 *}=((6.33,6.4,6.36),(6.19,6.24,6.49),(6.23,6.44,6.25),(6.91,6.81,6.71)) .
\end{gathered}
$$


Figure 1 shows that Algorithm 1 converges to a Nash equilibrium payoffs of both the firms after few iterations. The total CPU time to compute Nash equilibrium is 7.71 seconds.

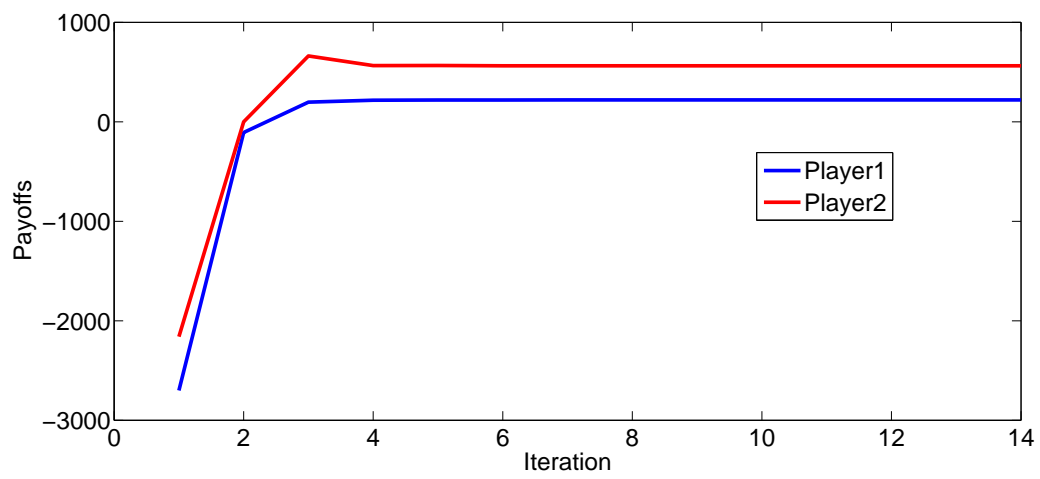

Fig. 1: Convergence of Nash equilibrium payoff

We also perform numerical experiments for a relatively large size model with 10 generation nodes and 12 distribution nodes. For this model, we take $\delta_{k j}=1, \beta_{k j}=\operatorname{randi}([30,50]), c_{k j}^{1}=\operatorname{randi}([12,18])$ and $c_{k j}^{2}=\operatorname{randi}([9,15])$ for all $k \in N$ and $j \in M$, where $\operatorname{randi}([a, b])$ generates a random integer number between closed interval $[a, b]$. For each $k \in N$ and $j \in M$, we choose $\varepsilon_{k j}^{1}$ and $\varepsilon_{k j}^{2}$ such that condition (33) is satisfied, and $\gamma_{k j}^{1}=\gamma_{k j}^{2}=50$. For $k \in N$ and $i=1,2$, we take the mean vector $\mu_{k}^{i}=0.15+\frac{1}{10} \operatorname{rand}(12,1)$ and the covariance matrix $\Sigma_{k}^{i}=B B^{T}+s \cdot I_{12 \times 12}$, where $B=\frac{1}{5} \operatorname{rand}(12,12)$ and $s>0$. The $\operatorname{rand}(12,1)$ generates a $12 \times 1$ random vector whose entries are between 0 and 1 and rand $(12,12)$ generates a $12 \times 12$ random matrix whose entries are between 0 and 1 . We consider 20 different instances of this model, and for each instance Algorithm 1 converges to a Nash equilibrium. The average time to compute a Nash equilibrium is 301.81 seconds. The Figure 2 represents the variation in the time for different instances.

\section{Conclusion}

We studied Nash equilibirum problem for the games with joint chance constraints, where the row vectors defining the random constraints are independent. We studied these games under distributionally robust framework. We considered a density based uncertainty set and four different types of twomoments based uncertainty sets. One of the moments based uncertainty sets is based on nonnegative support. For moments based uncertainty sets, we proposed a new convex reformulation of a distributionally robust joint chance constraint using logarithmic transformation. Under standard assumptions on 


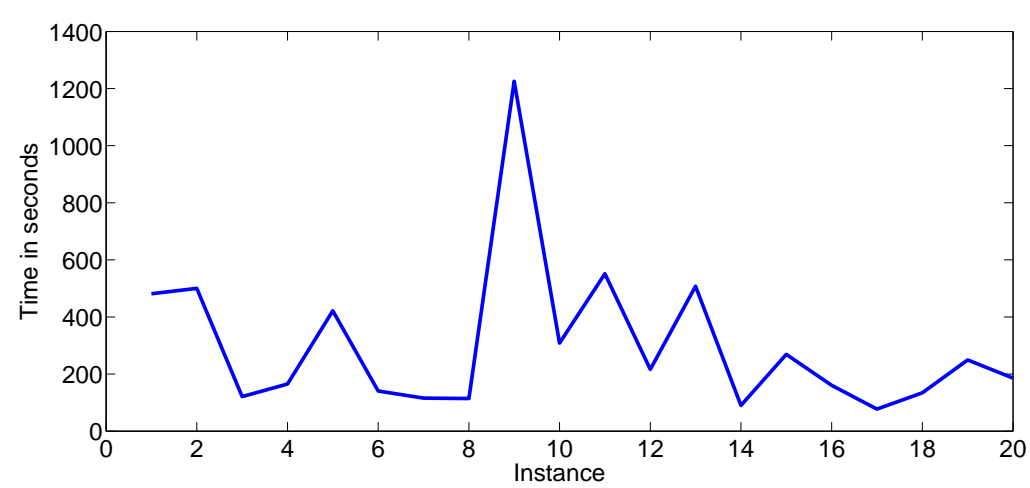

Fig. 2: Time vs Instances

players' payoff functions we showed that there exists a Nash equilibrium of a DRCCG. As an application of these games, we proposed a Cournot competition model in electricity market, which covers the generation and distribution of electricity. The best response algorithm is used to compute the Nash equilibria of various randomly generated instances of the game.

\section{Acknowledgement}

This research was supported by DST/CEFIPRA Project No. IFC/4117/DSTCNRS-5th call/2017-18/2 and CNRS Project No. AR/SB:2018-07-440.

\section{References}

1. Aghassi, M., Bertsimas, D.: Robust game theory. Mathematical Programming Series B 107, 231-273 (2006)

2. Basar, T., Olsder, G.J.: Dynamic noncooperative game theory, 2nd edn. SIAM, Philadelphia, PA (1999)

3. Blau, R.A.: Random-payoff two person zero-sum games. Operations Research 22(6), 1243-1251 (1974)

4. Calafiore, G.C., Ghaoui, L.E.: On distributionally robust chanceconstrained linear programs. Journal of Optimization Theory and Applications 130, 1-22 (2006)

5. Cassidy, R.G., Field, C.A., Kirby, M.J.L.: Solution of a satisficing model for random payoff games. Management Science 19(3), 266-271 (1972)

6. Charnes, A., Kirby, M.J.L., Raike, W.M.: Zero-zero chance-constrained games. Theory of Probability and its Applications 13(4), 628-646 (1968)

7. Cheng, J., Delage, E., Lisser, A.: Distributionally robust stochastic knapsack problem. SIAM Journal of Optimization 24(3), 1485-1506 (2014) 
8. Cheng, J., Leung, J., Lisser, A.: Random-payoff two-person zero-sum game with joint chance constraints. European Journal of Operational Research 251(1), 213-219 (2016)

9. Cheng, J., Lisser, A.: A second-order cone programming approach for linear programs with joint probabilistic constraints. Operations Research Letters 40(5), 325-328 (2012)

10. Conejo, A.J., Nogales, F.J., Arroyo, J.M., García-Bertrand, R.: Riskconstrained self-scheduling of a thermal power producer. IEEE Transactions on Power Systems 19(3), 1569-1574 (2004)

11. Debreu, G.: A social equilibrium existence theorem. Proceedings of National Academy of Sciences 38, 886-893 (1952)

12. El-Ghaoui, L., Oks, M., Oustry, F.: Worst-case value-at-risk and robust portfolio optimization: A conic programming approach. Operations Research 51(4), 543-556 (2003)

13. Fan, K.: Applications oi a theorem concerning sets with convex sections. Mathematische Annalen 163, 189-203 (1966)

14. Henrion, R.: Structural properties of linear probabilistic constraints. Optimization 56(4), 425-440 (2007)

15. Jadamba, B., Raciti, F.: Variational inequality approach to stochastic Nash equilibrium problems with an application to Cournot oligopoly. Journal of Optimization Theory and Application 165(3), 1050-1070 (2015)

16. Jiang, H., Shanbhag, U.V., Meyn, S.P.: Distributed computation of equilibria in misspecified convex stochastic Nash games. IEEE Transactions on Automatic Control 63(2), 360-371 (2018)

17. Jiang, R., Guan, Y.: Data-driven chance constrained stochastic program. Mathematical Programming 158, 291-327 (2016)

18. Kannan, A., Shanbhag, U.V., Kim, H.M.: Addressing supply-side risk in uncertain power markets: stochastic Nash models, scalable algorithms and error analysis. Optimization Methods and Software 28(5), 1095-1138 (2013)

19. Koshal, J., Nedić, A., Shanbhag, U.V.: Regularized iterative stochastic approximation methods for stochastic variational inequality problems. IEEE Transactions on Automatic Control 58(3), 594-609 (2013)

20. Liu, J., Lisser, A., Chen, Z.: Stochastic geometric optimization with joint probabilistic constraints. Operations Research Letters 44, 687-691 (2016)

21. Liu, J., Lisser, A., Chen, Z.: Distributionally robust chance constrained geometric optimization (2019). URL http://www.optimization-online. org/DB_FILE/2019/07/7290.pdf

22. Liu, Y., Xu, H., Yang, S.J.S., Zhang, J.: Distributionally robust equilibrium for continuous games: Nash and Stackelberg models. European Journal of Operational Research 265, 631-643 (2018)

23. Nash, J.F.: Equilibrium points in n-person games. Proceedings of the National Academy of Sciences 36(1), 48-49 (1950)

24. von Neumann, J.: On the theory of games. Math. Annalen 100(1), 295320 (1928) 
25. Peng, S., Singh, V.V., Lisser, A.: General sum games with joint chance constraints. Operations Research Letters 56, 482-486 (2018)

26. Ravat, U., Shanbhag, U.V.: On the characterization of solution sets of smooth and nonsmooth convex stochastic Nash games. SIAM Journal of Optimization 21(3), 1168-1199 (2011)

27. Rujeerapaiboon, N., Kuhn, D., Wiesemann, W.: Chebyshev inequalities for products of random variables. Mathematics of Operations Research 43(3), 887-918 (2018)

28. Shapiro, A.: Semi-infinite programming. chap. On Duality Theory of Conic Linear Problems, pp. 135-165. Springer, Boston, MA (2001)

29. Singh, V.V., Jouini, O., Lisser, A.: Existence of Nash equilibrium for chance-constrained games. Operations Research Letters 44(5), 640-644 (2016)

30. Singh, V.V., Jouini, O., Lisser, A.: Distributionally robust chanceconstrained games: Existence and characterization of Nash equilibrium. Optimization Letters 11(7), 1385-1405 (2017)

31. Singh, V.V., Lisser, A.: A characterization of Nash equilibrium for the games with random payoffs. Journal of Optimization Theory and Applications 178(3), 998-1013 (2018)

32. Singh, V.V., Lisser, A.: Variational inequality formulation for the games with random payoffs. Journal of Global Optimization 72, 743-760 (2018)

33. Singh, V.V., Lisser, A.: A second order cone programming formulation for zero sum game with chance constraints. European Journal of Operational Research 275, 839-845 (2019)

34. Xie, Y., Shanbhag, U.V.: On robust solutions to uncertain linear complementarity problems and their variants. SIAM Journal on Optimization 26, 2120-2159 (2016)

35. Xu, H., Zhang, D.: Stochastic Nash equilibrium problems: Sample average approximation and applications. Computational Optimization and Applications 55(3), 597-645 (2013)

36. Yousefian, F., Nedić, A., Shanbhag, U.V.: On stochastic mirror-prox algorithms for stochastic cartesian variational inequalities: Randomized block coordinate and optimal averaging schemes. Set-Valued and Variational Analysis 26, 789-819 (2018) 